\title{
Plataforma virtual: una herramienta didáctica para el Proceso de Enseñanza Aprendizaje ${ }^{1}$
}

Perla Cristina Guzzetti de Marecos

perlaguzzetti@hotmail.com

\section{RESUMEN}

FHyCE- UNP

Los cambios acelerados de la tecnología en la actualidad sugieren rever el enfoque de la educación y con ello la necesidad de replantear el proceso de enseñanza aprendizaje con un cambio en la estructura mental de los principales actores. Esta investigación describe los aspectos positivos y negativos en la implementación de plataformas virtuales como herramienta didáctica en el proceso de enseñanza aprendizaje. Es un estudio cualitativo y descriptivo basado en la revisión bibliográfica de artículos científicos sobre investigaciones producidas en países de América Latina (Colombia, Perú, Córdoba Argentina, Plan Nacional de Educación 2024 - Paraguay) y España. Se destaca que la plataforma virtual es un recurso didáctico válido en el ámbito educativo, que favorece el trabajo autónomo, innovación, motivación y comunicación con el docente; sin embargo, existen dificultades en su implementación como la conectividad a internet, costos, administración y recursos necesarios para el efecto. Se debe trabajar en la cultura del docente y estudiante para una mejor inserción en la era digital.

Palabras claves: aprendizaje; educación; estrategia; plataforma virtual

\footnotetext{
${ }^{1}$ Artículo realizado en el marco del doctorado en Ciencias de la Educación de la Facultad de Humanidades y Ciencias de la Educación de la Universidad Nacional de Pilar.
} 


\title{
Virtual platform: a didactic tool for the Teaching-Learning Process
}

\begin{abstract}
The accelerated changes in technology nowadays suggest a revision of the approach to education and with it the need to rethink the teaching-learning process with a change in the mental structure of the main actors. This research describes the positive and negative aspects in the implementation of virtual platforms as a didactic tool in the teachinglearning process. It is a qualitative and descriptive study based on the bibliographic review of scientific articles on research produced in Latin American countries (Colombia, Peru, Córdoba - Argentina, National Education Plan 2024 - Paraguay) and Spain. It is highlighted that the virtual platform is a valid didactic resource in the educational field, which favors autonomous work, innovation, motivation and communication with the teacher; However, there are difficulties in its implementation, such as internet connectivity, costs, administration and the necessary resources for this purpose. You must work in the culture of the teacher and student for a better insertion in the digital age.
\end{abstract}

Keywords: learning; education; strategy; virtual platform

Artículo recibido: 10 oct. 2020 Aceptado para publicación: 16 nov. 2020 Correspondencia:perlaguzzetti@hotmail.com, Conflictos de Interés: Ninguna que declarar 


\section{INTRODUCCIÓN}

Existe una preocupación a nivel mundial sobre la calidad de las metodologías de enseñanza y de los aprendizajes de los estudiantes a nivel universitario, lo que pone de relieve la necesidad de que estos últimos tengan un papel más activo en su proceso de aprendizaje y que sean capaces de aprender (Martínez Sarmiento \& Gaeta González, 2018).

Así también, Terigi (2015) expresa que en el nivel secundario se están afrontando grandes desigualdades; problemas de deserción y repitencia, por lo que es fundamental hacer foco en las condiciones en que se producen, las cuestiones vinculadas con el régimen académico, la estructura curricular, la asistencia, etc., que permitan definir trayectos más adecuados a las circunstancias de los estudiantes.

Según, Lugo y Ithurburu (2019) las agendas educativas de los países de América Latina incorporan acciones y programas que apuntan a incluir las tecnologías para la enseñanza y el aprendizaje. Así también, Paraguay, en el Plan Nacional de Educación 2024, en su apartado Educación, Ciencia y Tecnología, propone que la incorporación de las nuevas tecnologías al sistema educativo "aporte al mejoramiento de los procesos educativos a través del uso de las TIC y al desarrollo en todos los estudiantes de las competencias digitales necesarias para participar y contribuir activamente en la sociedad" (p. 17).

Lugo y Ithurburu (2019) expresan que los cambios planteados por las tecnologías manifiestan la caducidad de los dispositivos tradicionales de formación, alentando la implementación de nuevas estrategias que comienzan a ser exploradas (capacitación situada, comunidades de aprendizaje, redes abiertas de profesores, etc).

El cambio más estructural no tiene relación solo con pensar en los educadores desde una nueva óptica. También implica pensar en formadores que sean mucho más que facilitadores de tecnología, cuyo rol esté bajo una relación diferente con el conocimiento y donde el valor central ya no está en el acceso o dominio de un conocimiento en particular sino en la capacidad de desfragmentar y reconstruir conocimientos bajo nuevas combinaciones, formatos y canales. En fin, cambio de rol tanto de las personas como de las instituciones que tradicionalmente han administrado el conocimiento (Cobo, 2016).

\section{Plataforma virtual y sus virtudes}

En un marco de innovación y cambio, en el que se supone responder de forma integral a los requerimientos que impone una sociedad del conocimiento y a las nuevas necesidades 
del entorno educativo surgen las plataformas educativas virtuales. Por lo tanto, se hace énfasis en la "virtualidad". Virtual tiene que ver con imaginario o aparente ("imagen virtual", por ejemplo), y se opone a real y físicamente presente. Un entorno o un aula virtual sustituye de algún modo (puede que complementando) a las aulas presenciales. Los entornos virtuales pretenden crear un aula donde ésta no está disponible, o bien ampliar las potencialidades del aula real (Díaz Becerro, 2009).

Pons, Colás y González (2011) expresan que las primeras plataformas virtuales creadas como apoyo de los procesos educativos, en ámbitos universitarios, aparecen en Canadá, a mediados de la década de los noventa. BECTA (British Educational Communications and Technology Agency, 2008), la agencia que lidera el desarrollo y la implantación educativa de las TIC en el Reino Unido, ha acuñado el término learning plataform para describir el conjunto de hardware, software y servicios de apoyo necesarios para la actividad formativa. Estas herramientas constituyen un sistema integrado que abre nuevos entornos de formación, respecto a los tradicionales modelos educativos.

Así también, Silvio (2005) expresa que una plataforma educativa es un entorno de trabajo en línea donde se comparten recursos para trabajar a distancia o en forma semipresencial, la cual debe poseer unas herramientas mínimas para su funcionamiento, las que divide en: Herramientas de gestión de contenidos, comunicación y colaboración, seguimiento y evaluación, administración y complementarias citado en (Urzúa Osorio et al., 2018).

A su vez, (Prada Núñez, Gamboa, \& Hernández Suárez, 2019) exponen que una alternativa viable se encuentra en las plataformas basadas en software libre, como LRN, Dokeos, Claroline, Ganesha, Ilias, OpenUSS LMS, Sakai, Docebo LMS, Moodle, entre otras. Conforme a lo expuesto, (Olmos Migueláñez, Martínez Abad, Torrecilla Sánchez, \& Mena Marcos, 2014) en su investigación, mencionan que Moodle es una de las plataformas más completas y adecuadas para su implementación en la educación superior porque proporciona tres recursos esenciales: la posibilidad de suministrar contenidos y actividades en línea, evaluación interactiva y la interacción y comunicación flexible entre el maestro y los estudiantes, lo que mejoran los resultados del aprendizaje.

Así mismo, Moodle permite que el profesor pueda calificar todas las actividades según el parámetro que el mismo decida. El sistema calcula la calificación para el período elegido y el alumno puede tener así información continua sobre su progreso en el curso (Díaz Becerro, 2009). Con relación a este mismo tema (Peralta, 2015) expresa que se evita el 
cúmulo de trabajo, con la asignación y corrección en muchos casos de manera automática y con mayor facilidad para una evaluación objetiva.

Respecto a la implementación de nuevas tecnologías, en América Latina se cuenta con experiencias en la utilización de plataformas virtuales, en diferentes área y niveles educativos, donde se exponen resultados positivos para el aprendizaje. Algunas de estas investigaciones se presentan a continuación destacando aportes de las mismas, éstas son:

a) Formación de futuros docentes en Matemática realizado en Colombia; destaca que la Plataforma de Aprendizaje a Distancia (PLAD) es una estrategia didáctica que favorece el proceso de enseñanza, potencia el trabajo autónomo y las competencias investigativas, al tiempo que favorece la comunicación con el docente (Prada et al. 2019). b) Cocreando componentes adaptativos con estilos de aprendizaje, en educación superior en Colombia; la considera facilitadora de objetivos (González Palacios et al., 2019). C) En clases de ciencias, nivel secundario en Córdoba-Argentina, presenta resultados de participación activa de los estudiantes y producciones argumentativas con un grado de complejidad alto (Mermoud, Ordoñez, \& García Romano, 2017) entre otros.

En Paraguay la educación a distancia, en el ámbito universitario, es una realidad que tuvo su inicio en el año 2004, con los primeros cursos ofrecidos por la Universidad Autónoma de Asunción. Actualmente, la Universidad Nacional de Asunción, la Universidad Nacional de Pilar y otras de carácter privado, están incursionando en trabajos a distancia con el de las tic. A partir de entonces fue consolidándose con ofertas de cursos a distancia ofrecidos por varias instituciones, tanto públicas como privadas, las cuales la han ido incorporando a sus ofertas académicas, primero de grado y posteriormente de postgrado; ofreciendo actualmente numerosos cursos, tanto ya sean de grado, como de especialización, maestrías y doctorados (Albertini González, 2017).

Conforme a lo expuesto y mencionado por Albertini González (2017), se presenta la siguiente tabla que expone las universidades de carácter público y privado con cursos a distancias en los que se aplica la modalidad virtual. 
Tabla $\mathrm{N}^{\circ} 1$ Universidades en Paraguay y modalidad a distancia

\begin{tabular}{|c|c|c|}
\hline \multirow{2}{*}{ Carácter } & \multicolumn{2}{|c|}{ Universidades en Paraguay y modalidad a distancia } \\
\hline & Universidad & Cursos ofrecidos \\
\hline \multirow{5}{*}{ 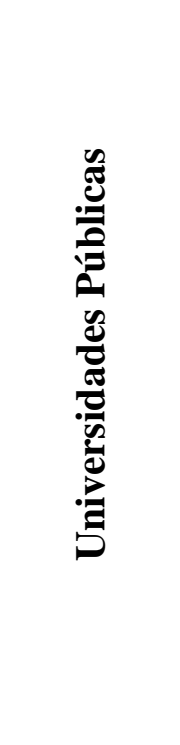 } & $\begin{array}{l}\text { Universidad Autónoma de } \\
\text { Asunción (UAA) }\end{array}$ & $\begin{array}{l}\text { Inicia cursos presenciales con } \\
\text { apoyo de e-learning (2004) }\end{array}$ \\
\hline & & $\begin{array}{l}\text { Incorpora cursos a distancia con } \\
\text { modalidad virtual (2008) }\end{array}$ \\
\hline & $\begin{array}{l}\text { Universidad Nacional de Asunción } \\
\text { (UNA) }\end{array}$ & $\begin{array}{l}\text { Ofrece cursos de grados y } \\
\text { especialidades a distancia }\end{array}$ \\
\hline & $\begin{array}{l}\text { Universidad Católica Nuestra } \\
\text { Señora de la Asunción }\end{array}$ & $\begin{array}{l}\text { Se encuentra en proceso de } \\
\text { adaptación de programas con } \\
\text { modalidad mixta }\end{array}$ \\
\hline & $\begin{array}{l}\text { Universidad Nacional de Pilar } \\
\text { (UNP) }\end{array}$ & $\begin{array}{l}\text { Ofrece carreras a distancia con } \\
\text { modalidad virtual }\end{array}$ \\
\hline 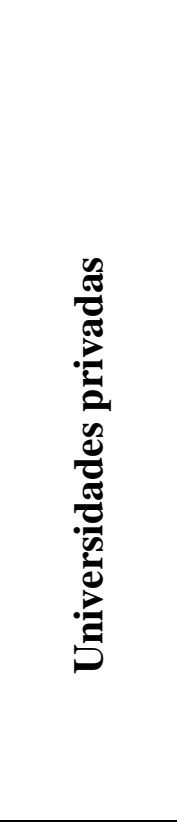 & $\begin{array}{l}\text { Universidad Americana, } \\
\text { Universidad Autónoma de } \\
\text { Asunción, Universidad Politécnica } \\
\text { y Artística del Paraguay - UPAP, } \\
\text { Universidad Iberoamericana, } \\
\text { Universidad Central del Paraguay, } \\
\text { Universidad Columbia del } \\
\text { Paraguay, Universidad de la } \\
\text { Integración de las Américas - } \\
\text { UNIDA, Universidad del Cono Sur } \\
\text { de las Américas - UCSA, } \\
\text { Universidad Privada del Guiará, } \\
\text { Universidad Técnica de } \\
\text { Comercialización y Desarrollo- } \\
\text { UTCD, Universidad Metropolitana } \\
\text { de Asunción, Universidad San } \\
\text { Lorenzo - UNISAL }\end{array}$ & Ofrecen cursos a distancia \\
\hline
\end{tabular}

Nota: Elaboración propia en base a la investigación denominada: Avances, Desafíos e

Impacto de la Educación Superior Virtual en Paraguay 2015 - 2017 (Albertini

González, 2017).

A pesar de las ventajas que proporciona, en Paraguay, la evaluación de la calidad de los cursos ofrecidos a distancia, está en fase de implementación, no existiendo hasta el 
momento ninguna institución universitaria, pública o privada, que cuente con certificación de calidad de los cursos on line (Albertini González, 2017).

\section{Experiencias positivas con la utilización de plataformas virtuales}

Según, Urzúa Osorio et al. (2018) algunas universidades que utilizan plataformas educativas para la entrega de trabajos escolares, les resulta una ventaja, ya que con la herramienta educativa no olvidaban los temas y fechas de entrega, era sustentable, no gastaban en hojas ni impresiones. Se comunicaban mediante foros o chat de la plataforma con los compañeros de clases y el docente.

La combinación entre el auge de la educación virtual y el uso cada vez mayor de los dispositivos móviles, por parte de los estudiantes, les genera un sentimiento de empoderamiento y se apropian de la información realizando una auténtica participación en la comunidad de aprendizaje, al sentirse responsable de su propio proceso educativo (Cantillo Valero, Roura Redondo, \& Sánchez Palacín, 2012).

Las TIC, en efecto, tienen el potencial de permitir el diseño de distintos escenarios de interacción, en experiencias significativas de ambientes de aprendizaje diversos. Cuando tales ambientes se han delineado con una intención clara y bien definida pueden resultar especialmente estimulantes en los procesos de aprendizaje (Prada et al., 2019).

Tal estímulo, mantiene a los estudiantes más motivados, el ambiente educativo gira en otro entorno, el cual está más familiarizado con la gran mayoría de los jóvenes de hoy día, considerando que cuando se trata de proyectos escritos, éstos, pueden incluir imágenes, colores, fondos, tan coloridos o variados como lo deseen de acuerdo con el tema desarrollado (Peralta, 2015).

La integración de las TIC en el aula conlleva un cambio en el rol del docente. El profesor plantea las actividades y propone secuencias didácticas que pueden desencadenar aprendizaje, tales como la búsqueda de información, el aprendizaje de nuevos conceptos, la publicación de las creaciones propias, el feedback de los otros participantes, o la revisión y mejora de los textos. El docente deja, por tanto, de ser un mero transmisor de conocimientos para desempeñar el rol de facilitador, o mediador de procesos de aprendizaje, cuya meta principal es transformar la información en conocimiento. Además, el profesor no sólo introduce al aprendiz a conceptos y datos sobre su área de conocimiento, sino que también le ayuda a desarrollar las habilidades cognitivas necesarias para poder comunicarse en el nuevo entorno (Díaz Becerro, 2009). 
Así también, Peralta (2015) sostiene que se fomenta una mayor responsabilidad en los estudiantes, ya que el tiempo asignado para toda práctica virtual tiene un cierre automático, y con penalización si así el profesor lo desea.

El aprendiz se constituye en constructor de conocimientos. Los conocimientos previos del alumno tienen una importancia fundamental, ya que constituyen la base sobre la que se integrarán los nuevos conocimientos. Los estudiantes realizan las tareas en base al conocimiento previo, conocimiento que se ve potenciado por la lectura de las intervenciones de los otros participantes. El profesor puede ayudar al aprendiz dándole feedback sobre su trabajo en sus interacciones a través de la plataforma educativa, y a través de los materiales didácticos que pone a su disposición en el aula virtual (andamiaje cognitivo), pero es el propio alumno el que va construyendo su conocimiento a través de las interacciones con otros estudiantes como él, con el profesor y con el material didáctico disponible (Díaz Becerro, 2009).

Según manifestaciones de alumnos, las plataformas virtuales ayudarían a evitar desplazamientos, compatibilizar el aspecto laboral con el estudio; una mayor oportunidad educativa; el acercamiento de la universidad a las personas distantes de ellas; evitaría la discriminación, disminución en los costos de estudio; a ser autodidactas, la disciplina personal; una mayor accesibilidad a la educación superior (Comet Weiler, 2016).

Los estudiantes no sólo son activos sino también actores, co-construyen el espacio virtual. En tal entorno de aprendizaje, estos pueden ser también diseñadores y productores de contenidos. En este sentido, el papel es mucho más participativo y activo, ya que puede contribuir con sus aportaciones, aumentar la base de conocimiento, reforzar enlaces, etc. En definitiva, el conocimiento es mucho más dinámico y cambiante. Al mismo tiempo, es preciso señalar que los entornos virtuales de aprendizaje no están restringidos a la enseñanza a distancia, también pueden enriquecer la enseñanza presencial (Silva Quiroz \& Romero, 2014).

Los entornos virtuales de aprendizaje reproducen el modelo de enseñanza/aprendizaje que tiene el profesor. Si su modelo es transmisor en el aula, en su virtualidad electrónica también lo será. La discusión con los alumnos, aprender a razonar y a ser convincente, la investigación en equipo, el trabajo cooperativo son aspectos que se pueden realizar en estos entornos (Díaz Becerro, 2009). 
Las plataformas virtuales, en este caso la plataforma virtual educativa, como herramienta; no solo permite hacer un trabajo acorde con los tiempos, sino un mejor uso y distribución del tiempo, permite retroalimentar el trabajo realizado por los estudiantes, evaluar un proyecto, un examen o pruebas sin la necesidad de una revisión física de cada asignación (Peralta, 2015).

En cada curso el profesor puede hacer uso de los diferentes módulos o herramientas que la plataforma integra para diseñar y organizar secuencialmente el proceso de aprendizaje. Todas las plataformas tienen una utilidad para la publicación de anuncios o avisos, una agenda o calendario del curso, un foro para realizar debates o realizar consultas, un generador de cuestionarios con preguntas de distintos tipos (elección múltiple, verdadero o falso, respuesta breve, etc.) o un módulo para que los alumnos envíen los trabajos, documentos o archivos que les solicite el profesor (Díaz Becerro, 2009).

Por ser un medio virtual a través del cual los estudiantes están en contacto permanente les es muy fácil tener acceso y poder avanzar con las tareas programadas y los proyectos asignados, así como llevar un control de sus avances y de sus debilidades en el proceso de aprendizaje (Peralta, 2015).

\section{Aspectos a considerar en la implementación de plataformas virtuales}

Los principales inconvenientes de las plataformas están relacionados con los elevados costos y la dificultad de su mantenimiento y administración. Así también, presenta limitaciones en su uso, relacionadas con problemas de conectividad, que los obliga a estar constantemente ingresando al sistema para verificar fechas de entrega o la asignación de nuevas actividades, puesto que no se cuenta con un sistema de alertas en donde se notifique la actualización de actividades por parte del docente (Prada et al., 2019).

Al respecto, uno de los obstáculos para el rápido avance de la educación virtual en la universidad paraguaya, es la baja penetración de internet en el país, que aunque haya estado creciendo constantemente en los últimos años, aún resulta insuficiente para encarar una expansión rápida y eficiente de la enseñanza a distancia en el ámbito universitario del país (Albertini González, 2017).

En contrapartida, González Palacios et al. (2019) sostienen que la dificultad más allá de lo técnico se encuentra en la falta de cultura tanto del docente como del estudiante, ambos deben entender y apreciar el concepto de educación virtual y usarlo en toda su extensión para potenciar lo que ya existe. Al respecto, Miño Puigcercós, Domingo Coscollola, \& 
Sancho Gil, (2019) expresan que las limitaciones no se encuentran en la metodología y los métodos implementados, sino en el desafío de introducir, en una cultura institucional tradicional, perspectivas de aprendizaje y prácticas de colaboración mejor desarrolladas en entornos no formales donde otras formas de aprendizaje, comunicación y relacionarse están permitidos y valorados.

Por tanto, el docente debe reflexionar sobre su praxis y tomar más en cuenta las diferencias individuales de los estudiantes, cumpliendo su papel de mediador del aprendizaje como promotor de la participación activa y reflexiva del educando. Es por ello que debe enseñar a sus estudiantes a discernir entre toda la gama de información que día a día debe confrontar, promoviendo en él y ella la búsqueda, el uso adecuado y proactivo y una actitud crítica y reflexiva que le permita tomar decisiones en relación con cualquier tópico (Márquez Marín, 2017).

En Paraguay, las barreras para la implementación de plataformas serían: el nivel socioeconómico; la existencia de muchos analfabetos informáticos; la accesibilidad limitada a los que tiene una computadora; el costo elevado del usufructo del Internet, el alcance limitado del Internet, el costo elevado del usufructo de ciber café, ; la creencia de que sólo lo presencial es válido o que el título no será válido para lo laboral; indiferencia por la formación, el temor a lo desconocido; la desconfianza hacia la validez del sistema; suspicacia hacia la capacidad de las instituciones que la implementan y la desconfianza en la propia capacidad (Comet Weiler, 2016).

Mascarell Palau, (2019) sostiene que la cultura digital en la que vivimos inmersos nos insta a incorporarnos de inmediato en el mundo de las tic, sin prejuicios, pero conscientes de hacer un uso racional y útil de las mismas, prevaleciendo el sentido didáctico sobre el tecnológico.

$\mathrm{Al}$ respecto, en Paraguay, tiene especial relevancia el hecho de que un elevado porcentaje de docentes universitarios, no son nativos digitales sino inmigrantes digitales, hecho que amplía las brechas generacional y digital existentes entre alumnos y profesores; lo cual a su vez exige un importante proceso de capacitación continua en el uso de las TICs (Albertini González, 2017).

Así mismo, los docentes de todos los niveles requieren de una formación específica en este campo, de una preparación adecuada con lo que de ellos se espera; de igual forma 
los profesores que tienen profesiones diferentes a la docencia, deben recibir instrucción en el uso técnico y pedagógico de los modernos medios (López Miño, 2017).

Al respecto López Miño, (2017) sostiene que el sistema educativo debe adaptarse a los cambios sociales y replantearse el papel que actualmente desempeñar el profesor, así como las competencias que debe poseer para desenvolverse en una sociedad de información. Las nuevas formas de enseñanza y de aprendizaje exigen habilidades como investigación, búsqueda, estudio, invención, adaptación, flexibilidad, creatividad, actitudes de tolerancia a la frustración para encontrar el uso pedagógico de la tecnología. Es necesario estar preparados para triunfos y fracasos, del docente y de sus alumnos, cada vez que se intente introducir una nueva tecnología.

Montes, (2015) expresa que la tendencia actual hacia la globalización, generalización y expansión del conocimiento da lugar a una transformación en las instituciones superiores, por lo que los profesionales deben ser capaces de mantener sus experiencias actualizadas a medida que se producen continuos avances en sus áreas de trabajo donde puedan utilizar nuevos métodos de enseñanza a través de las tecnologías de la información y la comunicación (TIC). Al respecto, se menciona a Melo Solarte y Díaz, (2018) quienes sostienen que los entornos virtuales de aprendizaje deben dejar de ser considerados escenarios fríos y rígidos, dedicados exclusivamente a compartir contenidos, realizar actividades y considerar las características que inciten o motiven al estudiante a realizar sus labores en pro de construir su conocimiento y desarrollar sus competencias. En cualquier caso, el problema más urgente es activar propuestas para reducir la distancia entre los entornos educativos formales y las "vidas digitales" (Scolari, Masanet, Guerrero Pico, \& Establés, 2018).

Según, Hernández Lara, Flores Barrios, Santiago Flores, \& Olarte García (2017) la gran incorporación de tecnologías de información y comunicación (TIC) a la educación abren un sinfín de posibilidades de adquisición de más y nuevos conocimientos. En este sentido, Leiva Guerrero y López Jiménez (2019) expresan que las plataformas virtuales permiten una formalización de las retroalimentaciones y acceso a las mismas en cualquier momento del proceso formativo, así como también conocer desde el inicio los criterios, rúbricas, fechas y protocolos que guían la elaboración de informes de cada instancia evaluativa de la práctica educativa. 


\section{ESTRATEGIAS METODOLÓGICAS O MATERIALES Y MÉTODOS}

En la investigación se empleó un estudio cualitativo y descriptivo, basado en la revisión bibliográfica de artículos científicos y discusión de autores, referentes a la implementación de la plataforma virtual en el proceso de enseñanza aprendizaje, en donde se visualizaron aspectos positivos y negativos; y experiencias a partir de la puesta en práctica de la misma en diferentes niveles educativos.

La investigación se apoyó en consultas de estudios previos que hayan analizado la implementación de plataformas virtuales y su impacto en la educación. Al respecto se utilizó como patrón de selección de la información documentos publicados en revistas nacionales e internacionales y documentos oficiales del MEC, a fin de contrastar de manera organizada los propósitos de estudios y aportes al tema.

En cuanto a la revisión de la información se empleó el siguiente criterio de búsqueda: a) Palabras claves: aprendizaje; educación; estrategia; plataforma virtual, b) Tipo de publicación: artículos, libros, revistas; c) Años de publicación: 2015-2019.

Una vez seleccionados los documentos, se importaron de la web y ordenaron en carpetas nominadas, primera, segunda y tercera revisión bibliográfica. Seguidamente, se procedió a señalar los resultados y principales aportes de cada material con el objeto de determinar si los hallazgos contribuían con el objetivo propuesto. En total se revisaron 46 artículos, en español e inglés, exceptuando autores y teorías clásicas, los cuales fueron publicados en revistas tecnológicas y educativas.

\section{RESULTADOS Y DISCUSIÓN}

Los resultados obtenidos permiten reconocer la efectividad de las plataformas virtuales como herramienta didáctica y así propiciar un espacio de aprendizaje que permita una interacción docente - alumno más dinámica y significativa, optimiza tiempo y recurso, por lo que se concuerda con Urzúa Osorio et al. (2018), ya que los estudiantes ahorrarían en fotocopias y materiales didácticos, como así también pueden realizar una lectura anterior a una clase presencial y con ello ahorrar tiempo para aclarar dudas y analizar temas de interés. Esto potenciaría el trabajo autónomo y las competencias investigativas. Sin embargo, estos resultados se obtendrán siempre que la conectividad a internet lo permita y el Estado potencie tal herramienta para que sea de mayor cobertura, ya que en Paraguay existe una baja penetrabilidad en la conectividad, aspecto en acuerdo con lo mencionado por Albertini González, (2017). 
Son varios los trabajos que han puesto de manifiesto que una de las barreras en la utilización de las tic va más allá de lo técnico, pues se encuentra en la falta de cultura tanto del docente como del estudiante, ambos deben entender y apreciar el concepto de educación virtual, tal como lo afirman los autores González Palacios et al. (2019) y Miño Puigcercós et al. (2019). Razón por la que se requiere de capacitaciones sobre la utilización y funcionamiento de dichas herramientas y así evitar el sesgo con la era digital. Esto sin olvidar, que una mentalidad abierta, el interés y la voluntad de aprender requiere de un proceso de cambios desde la concepción misma que tienen los diferentes actores sobre la educación.

Márquez, (2017) sostiene que los docentes en ejercicio requieren actualización continua y permanente que disminuya la tensión y el desconocimiento y les permitan enfrentar los cambios con apertura y dedicación. Al respecto y acotando a la idea, dicha actualización debe responder verdaderamente a una formación práctica en cuanto a la utilización de la tecnología, desde la implementación de dispositivos móviles, plataformas, redes, materiales audio visulaes, en el proceso de enseñanza aprendizaje y evitar la mera teoría, la cual, si bien es importante, poco colabora en el momento de la praxis educativa.

En cuanto al inconveniente de las plataformas virtuales relacionado a que no cuentan con un sistema de alertas en donde se notifiquen las actualizaciones de actividades por parte del docente, según Prada et al. (2019), se podrían ver otras alternativas para paliar la dificultad, como dispositivos móviles que pueden colaborar a través de mensajes en grupos enviados por el docente.

Se destaca la importancia de la implementación de nuevas estrategias educativas, la cual resulta imperiosa conforme a los cambios actuales en la tecnología. Al respecto, la formación del docente debe estar orientada a innovar y experimentar con las TIC, favoreciendo el desarrollo y la adquisición de diferentes competencias.

Finalmente, se coincide con la idea de incorporar acciones y programas que apuntan a incluir las tecnologías para la enseñanza y el aprendizaje, la cual comparten los autores; Lugo y Ithurburu (2019), Cobo, (2016), en Paraguay, el Plan Nacional de Educación 2020, ya que hoy día la tecnología avanza a pasos agigantados y los alumnos no están ajenos a dicha realidad, se actualizan y la utilizan en sus actividades cotidianas, por lo que es necesario que los docentes renueven su bagaje de estrategias de enseñanza, las adecue a las nuevas tendencias y empleen las tecnologías como herramienta didáctica y 
así propiciar un espacio de aprendizaje que permita una interacción docente - alumno más dinámica y significativa.

\section{CONCLUSIÓN O CONSIDERACIONES FINALES}

Las instituciones educativas que utilizan plataformas virtuales como herramienta didáctica en el proceso de enseñanza y aprendizaje presentan experiencias favorables, las cuales apuntan hacia resultados óptimos, logros de objetivos y con espacios de interrelación significativos, creativos, innovadores y motivadores para los estudiantes.

El modelo de entorno virtual mediado por las tecnologías requiere que el estudiante construya y reconstruya sus capacidades básicas cognitivas, a partir de sus intereses, experiencias, expectativas, conocimientos, que le permitan adquirir su propio aprendizaje con una metodología de trabajo particular y disciplinada.

Así también, pueda incorporar en su diario vivir redes de conocimiento, y de esta forma ejercer libertad y apropiación de ciencias, fortaleciendo el statu quo de su aprendizaje (Abril Lancheros, 2018).

Partiendo de lo mencionado por Huss (2019), quien concluye que en el siglo XXI si los maestros no se están preparando para enseñar en un aula digital, o al menos con una entrega de currículum mejorada digitalmente, entonces sus habilidades están desactualizadas incluso antes de comenzar su profesión de educador; cabe mencionar que, es menester de las autoridades educativas incorporar capacitaciones actualizadas sobre las tic a fin de contar con docentes competentes que conduzcan eficazmente la educación del país hacia un rango de calidad y abierta a la era tecnológica.

La implementación de plataformas virtuales en el proceso de enseñanza, resulta una herramienta válida, innovadora y eficaz para el logro de aprendizajes significativos en la cultura digital presente en la actualidad.

Es preciso considerar que los estudiantes no solo ahorrarán papeles, recordarán fechas de entrega, contarán con materiales diversos, según Urzúa Osorio et al. (2018) sino que también, podrán plasmar sus ideas y aprender de una manera creativa, conforme a sus intereses y tiempos, a modo de construir aprendizajes significativos.

En cuanto a aspectos negativos, la implementación de esta herramienta de enseñanza aprendizaje puede verse afectada por cuestiones técnicas, de conectividad, considerando la baja cobertura de la misma en nuestro país y la cultura, específicamente de los docentes y alumnos quienes requieren de capacitaciones permanentes y una mentalidad abierta 
basada en el interés y la voluntad de aprender y actualizarse conforme a la nueva era digital.

Así también, la carencia de recursos y los costos elevados, ya sean de aparatos electrónicos, administrativos e internet, específicamente en Paraguay, dificultan en gran medida la implementación y puesta en práctica de estrategias educativas virtuales destinadas a todos los estudiantes y docentes, sin exclusión alguna y de manera eficaz.

\section{LISTA DE REFERENCIAS}

Abril Lancheros, M. S. (2018). Motivación del aprendizaje en línea. Revista Panorama, Vol.12. $\mathrm{N}^{\circ} 22.43-56$.

Albertini González, F. A. (2017). Avances, Desafios e Impacto de la Educación Superior Virtual en Paraguay 2015 - 2017 . ScientiAmericana, 1-23 .

Cantillo Valero, C., Roura Redondo, M., \& Sánchez Palacín, A. (2012). Tendencias actuales en el uso de dispositivos móviles en educación. La Educación, 1-21. N"147.

Comet Weiler, C. (2016). Percepcioón de los estudiantes universitarios sobre la incidencia de los factores socioculturales enn la implementación de la tefeformación en Paraguay. ACADEMO Revista de Investigación en Ciencias Sociales y Humanidades, 1-24 Vol.3 $\mathrm{N}^{\circ} 1$.

Díaz Becerro, S. (2009). Introducción a las plataformas virtuales en la enseñanza . Revista digital para profesionales de la enseñanza, 1-7. $\mathrm{N}^{\circ} 2$.

González Palacios, L., Cuatindioy, J., González Palacio, M., Luna, M., Ortiz Pabón, H. J., \& Bedoya, J. A. (2019). Cocreando componentes adaptativos para una plataforma virtual basados en estilos de aprendizaje Cocreation of adaptative components for a virtual platform based on learning styles . 14th Iberian Conference on Information Systems and Technologies (CISTI) .

Hernández Lara, J. L., Flores Barrios, L., Santiago Flores, M., \& Olarte García, M. (2017). PLATAFORMA VIRTUAL Y SU EFICIENCIA EN EL APRENDIZAJE EN ALUMNOS UNIVERSITARIOS . Academia Journals, Vol. 9. $\mathrm{N}^{\circ}$ 4. 23012305.

Huss, R. (2019). Preparing Teachers for the 21st Century Classroom. Internet Learning Journal, Vol.7. $\mathrm{N}^{\circ}$ 1. 15-29. 
Leiva Guerrero, M. V., \& López Jiménez, T. (2019). Uso de plataforma virtual para retroalimentar la formación del profesorado. Pensamiento Educativo, Vol. $56 \mathrm{~N}^{\circ}$ 2.1.17.

López Miño, A. T. (2017). Educación en la sociedad de la tecnología de la educación. Vinculando, 1-7.

Lugo, M. T., \& Ithurburu, V. (2019). Políticas digitales en América Latina. Tecnologías para fortalecer la educación de calidad. Revista Iberoamericana de Educación [(2019), vol. 79 núm. 1, pp. 11-31], vol.79, $\mathrm{N}^{\circ} 1,11-31$.

Márquez Marín, J. C. (2017). Tecnologías de la información en la educación Venezolana. Vinculando, 1-13.

Martínez Sarmiento, L. F., \& Gaeta González, M. L. (2018). Utilización de la plataforma virtual Moodle para el desarrollo del aprendizaje autorregulado en estudiantes universitarios. Educar, vol. 55/2 479-498.

Mascarell Palau, D. (2019). IMPLEMENTATION AND USE OF ICT. MOBILE DEVICES IN EDUCATION IN ARTS. A STATE OF AFFAIRS (IMPLEMENTACIÓN Y USO DE LAS TIC. DISPOSITIVOS MÓVILES EN EDUCACIÓN EN ARTES. UN ESTADO DE LA CUESTIÓN). Revista de Comunicación de la SEECI, $\mathrm{N}^{\circ}$ 50. 73-86.

Melo solarte, D. S., \& Díaz, P. A. (2018). El Aprendizaje Afectivo y la Gamificación en Escenarios de Educación Virtual. Información Tecnológica, Vol. 29 N³. 237 248.

Mermoud, S. R., Ordoñez, C., \& García Romano, L. (2017). Potencialidades de un entorno virtual de aprendizaje para argumentar en clases de ciencias en la escuela secundaria . Revista Eureka sobre Enseñanza y Divulgación de las Ciencias, Vol.14 (3), 587-600.

Miño Puigcercós, R., Domingo Coscollola, M., \& Sancho Gil, J. M. (2019). TRANSFORMING THE TEACHING AND LEARNING CULTURE IN HIGHER EDUCATION FROM A DIY PERSPECTIVE (TRANSFORMAR LA CULTURA DE ENSEÑANZA Y APRENDIZAJE EN LA EDUCACIÓN SUPERIOR DESDE UNA PERSPECTIVA DIY). Educación XX1, Vol.22. ํำ. 139-160. 
Montes, A. M. (2015). OPTIMIZACIÓN DE LA AUTOFORMACIÓN A TRAVÉS DE LA ENSEÑANZA VIRTUAL . Panorama, Vol.9. N⒘ 41-49.

Olmos Migueláñez, S., Martínez Abad, F., Torrecilla Sánchez, E. M., \& Mena Marcos, J. (2014). PSYCHOMETRIC ANALYSIS OF A PERCEPTION SCALE ON THE USEFULNESS OF MOODLE IN THE UNIVERSITY [Análisis psicométrico de una escala de percepción sobre la utilidad de Moodle en la Universidad] . Revista ELectrónica de Investigación y Evaluación Educativa, vol. 20(2), 1-18.

Peralta, W. M. (2015). La plataforma virtual como herramienta de enseñanza. Vinculando, 1-8.

Plan Nacional de Educación 2024. Hacia el centenario de la escuela nueva de Ramón Indalecio Cardozo. Disponible en http://.becal.gov.py > uploads > 2. Plan Nacional de Educacion 2024.pdf

Prada Núñez, R., Gamboa, A., \& Hernández Suárez, C. (2019). Usos y efectos de la implementación de una plataforma digital en el proceso de enseñanza de futuros docentes en matemáticas. Proceso de enseñanza| Revista virtual, Vol 57, 137156.

Scolari, C. A., Masanet, M. J., Guerrero Pico, M., \& Establés, M. J. (2018). TRANSMEDIA LITERACY IN THE NEW MEDIA ECOLOGY: TEENS' TRANSMEDIA SKILLS AND INFORMAL LEARNING STRATEGIES (Alfabetismo transmedia en la nueva ecología de medios. Competencias transmedia y estrategias de aprendizaje informal de los adolescentes). El profesional de la información, 2018, julio-agosto, v. 27, n. 4. eISSN: 1699-2407 801, Vol. 27. $\mathrm{N}^{\circ}$ 4. 801-812.

Silva Quiroz, J. E., \& Romero, M. (2014). La virtualidad una oportunidad para innovar en educación: un modelo para el diseño de entornos virtuales de aprendizaje. Didasc@lia: Didáctica y Educación, Vol.V. º1. 1- 22.

Urzúa Osorio, D., Bedolla Solano, I., Pacheco Salazar, E., Astudillo Hernández, C., García Lozano, E., \& López Cortez, A. (2018). ANÁLISIS Y DISEÑO DE UNA PLATAFORMA ESTANDARIZADA PARA LA EDUCACIÓN Y EVALUACIÓN DE COMPETENCIAS EN EL INSTITUTO TECNOLÓGICO NACIONAL CAMPUS ACAPULCO . Academia Journals, Vol. 10, No. 8, 56905695. 
